\title{
Una revisión de la literatura acerca de las características neuropsicológicas de niños con craneosinostosis simple en diferentes edades
} A review of the literature on the neuropsychological
characteristics of children with single suture craniosynostosis
at different ages

Julieta Moreno V. ${ }^{1}$, Guillermina Yáñez T. ${ }^{2}$, Belén Prieto C. ${ }^{2}$, Yaneth Rodríguez A. ${ }^{3}$ y Antonio García M. ${ }^{4}$

Introduction: Single suture craniosynostosis is the premature closure of one or more cranial sutures. It leads to abnormal skull growth and in some cases to structural and/or functional brain abnormalities. If these anomalies cause a psychomotor development lag and/or cognitive impairment is a controversial topic. This study aims to review the main findings related to psychomotor development and cognitive functioning in children with craniosynostosis, from birth to school age, dividing them into three stages: birth to 3 years, preschool and school. Method: An electronic search was made in PubMed from 2000 to 2015, using the following keywords: "craniosynostosis and neurodevelopment", "craniosynostosis and neuropsychological", "craniosynostosis and cognition". Results: In the early stage of life some children with craniosynostosis can have motor, language and cognition deficits; these impairments are also present in a variable percentage of preschool children, in some cases accompanied with behavior disorders; deficits in attention, executive functions and learning disorders, are reported in school years. Conclusion: Some neuropsychological alterations are found in a variable proportion of patients with craniosynostosis in all stages of development whichare revealed depending on the demands of each age. It is difficult to draw a cognitive profile for this population because of the heterogeneity related to characteristics of the sample, time of assessment, functions assessed and instrument used. It is important to make longitudinal neuropsychological assessments to know how children with craniosynostosis are developing in late stages and to develop consensus about cognitive functions and assessment instruments appropriate for each age.

Key words: Single suture craniosynostosis, cognitive impairment, neuropsychology. Rev Chil Neuro-Psiquiat 2017; 55 (1): 52-63

Recibido: 06/10/2016

Aceptado: 03/02/2017

Los autores no presentan ningún tipo de conflicto de interés.

Fuentes de financiamiento: Julieta Moreno V. es becaria CONACYT (261233) para la realización de estudios de Doctorado en Psicología.

Alumna de Doctorado en Psicología. FES Iztacala, Universidad Nacional Autónoma de México, Estado de México, México. Proyecto de Neurociencias de la UIICSE. FES Iztacala, Universidad Nacional Autónoma de México, Estado de México, México.

3 Departamento de Neuropsicología del Instituto Nacional de Neurología y Neurocirugía, Ciudad de México, D.F., México. 4 Jefe del Servicio de Neurocirugía Pediátrica de la Unidad Médica de Alta Especialidad del Hospital General de Centro Médico Nacional La Raza, Ciudad de México, D.F., México. 


\section{Introducción}

\section{Definición y clasificación de la craneosinostosis}

La craneosinostosis es el resultado de la fusión prematura de las suturas craneales ${ }^{1-6}$. Éstas son fibras densas que conectan tejido intramembranoso formado por huesos, cuya función es permitir el paso a través del canal del parto, servir como amortiguadores, permitir el crecimiento del cerebro y prevenir la separación de los huesos. Las suturas craneales son: metópica, coronal, sagital y lambdoidea. Como resultado de este cierre, el crecimiento del cráneo se detiene en dirección perpendicular de la sutura cerrada y hay una sobre expansión compensatoria en las suturas abiertas ${ }^{4}$.

Cohen $^{7}$, propuso tres dicotomías para clasificar a las craneosinostosis: simple o compuesta, primaria o secundaria y aislada o sindromática. La craneosinostosis simple es cuando sólo una sutura se fusiona y compuesta cuando lo hacen dos o más suturas. Respecto a la segunda dicotomía, la craneosinostosis primaria es la más común y en ésta no hay una causa identificable que la origine, mientras que la secundaria es provocada por otro trastorno. Finalmente, la craneosinostosis aislada se diagnostica cuando el paciente no tiene otra anormalidad, y es sindromática cuando ocurre con otros defectos morfológicos.

Van Veelen-Vincent et al. ${ }^{2}$ agrupan las craneosinostosis primarias no sindromáticas de acuerdo al número de suturas involucradas: única o múltiples. El nombre que reciben es de acuerdo a la forma resultante del cráneo según la sutura afectada: escafocefalia, cuando el cráneo adopta una forma alargada y la región parietal está estrecha en sentido transversal y generalmente adopta esta forma cuando la sutura sagital está comprometida; cuando el cráneo adopta una forma asimétrica, el lado afectado presenta un aplanamiento de la frente y un desplazamiento lateral de la raíz nasal, se le denomina plagiocefalia y ésta puede ser derecha o izquierda, dependiendo de qué sutura coronal esté fusionada; una frente estrecha y en forma de piso como la proa de un barco se denomina trigonocefalia y generalmente se da cuando la sutura metópica se fusiona prematuramente; una asimetría facial se denomina plagiocefalia posterior que involucra la fusión de la sutura lambdoidea. Las craneosinostosis múltiples son: braquicefalia que es cuando se observa aplanamiento de la parte baja de la frente mientras que la parte superior tiende a abombarse, involucra la fusión de toda la sutura coronal; la oxicefalia, cuando las suturas bicoronal y sagital están comprometidas; y turricefalia, que involucra ambas suturas coronales y el cráneo tiene una forma de torre ${ }^{8}$.

\section{Alteraciones estructurales y funcionales asociadas a la craneosinostosis simple}

Recientemente se discute si una anomalía craneal puede condicionar una alteración estructural y/o funcional del sistema nervioso central. En algunos pacientes con craneosinostosis simple, en estudios radiológicos, se han encontrado anomalías en el espacio subaracnoideo por debajo de la sutura fusionada, así como compresión del sistema ventricular ${ }^{9}$ lo que podría sugerir que el tejido cortical y/o subcortical, se comprime en el proceso de crecimiento dentro de un cráneo con capacidad limitada. Magge et al. ${ }^{10}$, encontraron en un estudio prequirúrgico con tomografía axial computarizada que más del $50 \%$ de un grupo de 80 pacientes con craneosinostosis presentaron algún hallazgo anormal, el más frecuente fue hidrocefalia externa benigna (45\%), que podría ocasionar un hematoma subdural.

En un estudio donde se comparó la resonancia magnética de 21 pacientes con las de niños sanos, se observó que los pacientes con plagiocefalia coronal derecha presentaron una morfología cortical y subcortical diferente de la de niños sanos respecto a las distancias entre estructuras corticales y subcorticales (p.e. reducción de la distancia entre los polos frontales y amígdala, tálamo y núcleo caudado, y reducción de la distancia entre los polos occipitales y el surco superior frontal y el cuerno anterior del ventrículo lateral $)^{11}$. Se ha reportado que, de una muestra de 121 pacientes con craneosinostosis simple, un bajo porcentaje (15\%) presentan alteraciones intracraneales, siendo la más frecuente malformación Chiari tipo I (más de la mitad de los 
pacientes que presentaron alteraciones) sobre todo en escafocefalia, cuatro casos con anormalidades en las regiones selar y paraselar, así como 2 casos de agenesia parcial del cuerpo calloso ${ }^{12}$.

Además, de las alteraciones estructurales, también se han encontrado algunas alteraciones funcionales. Cohen y Persing ${ }^{13}$ realizaron una revisión y refieren que la hipertensión intracraneal es el efecto negativo más común en esta patología. Se cree que la hipertensión promueve la hipovascularización en la región próxima de la sutura fusionada y que, a su vez, genera hipoplasia del tejido cerebral subyacente. Aunque la hipertensión craneal es más común en las craneosinostosis sindromáticas, mientras que en las craneosinostosis simples se manejan distintas frecuencias, por ejemplo, Tamburrini et al. ${ }^{14}$ sugieren que varía entre el 4 y el $20 \%$, por su parte Van Veelen-Vincent et al. ${ }^{2}$ mencionan que en pacientes con braquicefalia $y$ craneosinostosis complejas la incidencia de hipertensión intracraneal se encuentra entre 30 y $60 \%$. El número de suturas afectadas y la edad son factores que influyen en el aumento de la hipertensión intracraneal ya que ésta aumenta conforme existen más suturas afectadas y la hipertensión es el doble de frecuente después de un año; principalmente en la escafocefalia y plagiocefalia, su frecuencia es incluso del cuádruple después de un año ${ }^{6}$, por lo que uno de los objetivos para la realización de la intervención quirúrgica temprana es prevenir este efecto.

David et al. ${ }^{15}$, usando la tomografía por emisión de positrones, en 10 niños con craneosinostosis sagital, coronal ometópica, encontraron un cambio en el metabolismo sobre todo en la región occipital que mejoró después de la cirugía. Con el uso de doppler transcraneal, realizando una comparación pre y postquirúrgica en 10 pacientes con craneosinostosis tanto sindrómicas como simples, se reportó que después de la cirugía de corrección aumentó el flujo sanguíneo ${ }^{16}$. Por su parte, Beckett et al. ${ }^{17}$ reportaron que 8 adolescentes postoperados de escafocefalia presentaron alteraciones en las conexiones de la corteza prefrontal, el lóbulo parietal superior y los giros angular y supramarginal en una comparación de grupos.
También se han reportado alteraciones sensoriales en los pacientes con craneosinostosis. En un estudio se documentó que el $84 \%$ de 38 pacientes con craneosinostosis que fueron evaluados presentaron alteraciones visuales que involucran anormalidades en los movimientos oculares y en campos visuales ${ }^{18}$. Vasco et al. ${ }^{19}$ encontraron que el $16 \%$ de 29 niños con craneosinostosis simple que evaluaron, presentaron una función visual normal (comportamiento oculomotor, agudeza visual, campos visuales y cambios en la fijación) antes de la cirugía y 12 meses después de la corrección quirúrgica el porcentaje aumentó a $65 \%$. Las mayores anomalías en el comportamiento oculomotor se presentaron en pacientes con plagiocefalia.

En lo que respecta al procesamiento auditivo, Hashim et al. ${ }^{20}$, usando potenciales relacionados con eventos encontraron que 15 niños con craneosinostosis sin cirugía presentaron menor amplitud en el componente P150, en comparación con un grupo control de niños sanos, lo que relacionaron con una menor respuesta cortical a la estimulación del lenguaje. Los autores mencionan que lo anterior podría ser el precursor del retraso en la adquisición del lenguaje reportado en la literatura.

La posibilidad de que las anomalías craneales puedan condicionar alteraciones cerebrales estructurales y funcionales ha generado diversa investigación al respecto y planteado también la probabilidad de que éstas pudieran estar condicionando a su vez alteraciones cognoscitivas y en el desarrollo psicomotor, una serie de investigaciones se han enfocado en estudiar uno o más de estos procesos en diferentes etapas del desarrollo. Este trabajo tiene como objetivo hacer una revisión de los principales hallazgos reportados con relación al funcionamiento cognoscitivo y del desarrollo desde el nacimiento hasta la edad escolar dividiéndolos en tres etapas: del nacimiento a 3 años, preescolar y escolar

\section{Método}

Se realizó una búsqueda electrónica en la base de datos PubMed utilizando las siguientes palabras 
clave: "craniosynostosis and neurodevelopment", "craniosynostosis and neuropsychological" y "craniosynostosis and cognition". Se seleccionaron artículos desde el año 2000 hasta el 2015 para realizar el análisis de la literatura existente relacionada con aspectos neuropsicológicos de pacientes con craneosinostosis.

\section{Resultados}

\section{Hallazgos neuropsicológicos en pacientes con craneosinostosis simple:}

\section{Del nacimiento a los 3 años de edad}

La mayor cantidad de trabajos en donde se evalúa a pacientes con craneosinostosis simple se enfoca en niños con edades del nacimiento a los 3 años. Los resultados de estos estudios son inconsistentes. Algunos autores refieren índices normales en el desarrollo cognoscitivo ${ }^{21-23} \mathrm{y}$ de forma más específica Toth et al..$^{22}$ encontraron que la memoria de trabajo y la inhibición no se ven afectadas en niños que ya fueron sometidos a cirugía en comparación con niños sanos.

Otras investigaciones reportan deficiencias entre las que se encuentran: retraso psicomotor ${ }^{21,24-30}$, $\operatorname{cognoscitivo~}^{25-31}$ y de lenguaje ${ }^{23,28-30}$. Es relevante mencionar que la mayoría de los estudios que mencionan deficiencias en niños con craneosinostosis carecen de grupo control (e. $\mathrm{g}^{25-29}$ ), sólo son referidos a normas, por lo que sería importante conocer si los déficits encontrados persisten cuando se compara el desempeño con un grupo control pareado.

Específicamente en evaluaciones realizadas prequirúrgicamente, en lo que respecta al lenguaje, Warschausky et al. ${ }^{23}$ encontraron que el $43 \%$ de la muestra evaluada tenían una calificación de una desviación estándar por debajo de la población normativa, una cifra similar a la que reportan Kapp-Simons et al. ${ }^{28}$ (45\%). Por su parte, Da Costa et $a l .{ }^{26}$ mencionan que los índices mental y motor de niños con craneosinostosis son más bajos que lo esperado por el grupo normativo, aunque las puntuaciones aún caen dentro de los rangos nor- males; en sentido opuesto, Kapp-Simons et al. ${ }^{28}$ encontraron que el $16 \%$ y el $38 \%$ de su muestra obtuvo puntuaciones por debajo de una desviación estándar en el índice mental y psicomotor. Speltz et $\mathrm{al}^{31}$ en una comparación con un grupo control encontraron diferencias estadísticamente significativas en los índices que evalúan las Escalas Bayley. También se ha mencionado que esta población tiene 2,2 veces más riesgo de presentar retraso psicomotor $^{26}$. Los detalles de las investigaciones que tienen evaluaciones prequirúrgicas se resumen en la Tabla 1. Cuando se realizan evaluaciones pre y postquirúrgicas, algunos estudios ${ }^{24,25,29}$ reportan mejoría en las evaluaciones posteriores a la cirugía sobre todo en la motricidad, otros estudios a pesar de la realización de la cirugía siguen reportando déficits en comparación con niños sanos ${ }^{27,30}$ (Tabla 2).

\section{Etapa preescolar}

La mayoría de estudios que evalúan el funcionamiento cognoscitivo de niños con craneosinostosis en etapa preescolar mencionan índices o puntajes globales. Igual que en la etapa anterior, también se encuentran resultados contradictorios (Tabla 3). Shipster et al..$^{32}$ observaron funcionamiento cognoscitivo normal, mientras que otros reportan alteraciones en el lenguaje ${ }^{32-36}$, en la cognición $^{33,36}$, en la motricidad ${ }^{36}$ y en la conducta ${ }^{33}$. El hallazgo más frecuente es el déficit en el lenguaje, los distintos estudios mencionan porcentajes variables de pacientes con estas alteraciones, desde el $30 \% \%^{33,35}$ hasta el $50 \%^{32,34}$. También se menciona que los pacientes con craneosinostosis simple tienen de 1,5 a 2 veces mayor probabilidad de presentar alteraciones cognitivas, de lenguaje y motoras que en la población normal ${ }^{36}$.

La mayoría de los estudios que mencionan el funcionamiento cognoscitivo en esta edad no tienen grupo control ${ }^{32-35}$ y algunos las muestras son pequeñas para establecer una relación causal entre las alteraciones encontradas y la craneosinostosis $^{34,35}$, además el estudio de Mendonca ${ }^{35}$ no establece el instrumento que utilizó para evaluar el lenguaje. 
Tabla 1. Estudios realizados sobre el funcionamiento cognoscitivo prequirúrgico durante los primeros 3 años de vida en pacientes con craneosinostosis simple

\begin{tabular}{|c|c|c|c|c|}
\hline Autores & $\begin{array}{l}\text { n de pacientes/diagnóstico/ } \\
\text { controles }\end{array}$ & $\begin{array}{l}\text { Edad en evaluación } \\
\text { prequirúrgica }\end{array}$ & Instrumentos & Resultados prequirúrgicos \\
\hline $\begin{array}{l}\text { Panchal } \\
\text { et al. }{ }^{21}\end{array}$ & $\begin{array}{l}21 / \mathrm{CS} \\
11 \text { escafocefalia } \\
5 \text { trigonocefalia } \\
2 \text { braquicefalia } \\
3 \text { plagiocefalia } \\
42 \text { con plagiocefalia sin sinostosis }\end{array}$ & 10,9 meses & BSID-I & $\begin{array}{l}\text { Sin DES en el índice mental. } \\
\text { Déficit motor }\end{array}$ \\
\hline $\begin{array}{l}\text { Kapp-Simon } \\
\text { et al. }{ }^{28}\end{array}$ & $\begin{array}{l}100 / \\
49 \text { escafocefalia } \\
24 \text { trigonocefalia } \\
10 \text { plagiocefalia derecha } \\
8 \text { plagiocefalia izquierda } \\
9 \text { plagiocefalia posterior }\end{array}$ & 7,3 meses & BSID-I, PLS & $\begin{array}{l}\text { DES en los índices mental, } \\
\text { psicomotor y la prueba de } \\
\text { lenguaje en comparación con } \\
\text { las normas de la prueba }\end{array}$ \\
\hline $\begin{array}{l}\text { Warschausky } \\
\text { et al. }{ }^{23}\end{array}$ & $22 /$ trigonocefalia & 10,6 meses & $\begin{array}{l}\text { Índice mental } \\
\text { de BSID-II }\end{array}$ & $\begin{array}{l}\text { Índice de desarrollo mental } \\
\text { normal. } 43 \% \text { coeficiente } \\
\text { de lenguaje debajo de una } \\
\text { desviación estándar }\end{array}$ \\
\hline $\begin{array}{l}\text { Speltz } \\
\text { et al. }{ }^{31}\end{array}$ & $125 / \mathrm{CS}$ & 2-24 meses & BSID-II y PLS & $\begin{array}{l}\text { Índice mental y motor } \\
\text { significativamente más bajos }\end{array}$ \\
\hline $\begin{array}{l}\text { Da Costa } \\
\text { et al. }{ }^{26}\end{array}$ & $\begin{array}{l}56 / \\
26 \text { escafocefalia } \\
20 \text { trigonocefalia } \\
10 \text { unicoronal }\end{array}$ & 4-16 meses & BSID-II & $\begin{array}{l}\text { Puntuaciones más bajas de lo } \\
\text { esperado en población general } \\
\text { en índices cognitivo y motor }\end{array}$ \\
\hline
\end{tabular}

CS: Craneosinostosis simple; BSID-I: Escalas Bayley del Desarrollo Infantil-I BSID-II: Escalas Bayley del Desarrollo Infantil II; DES: Diferencias estadísticamente significativas; PLS: Preschool Language Scale.

\section{Etapa escolar}

Cuando los estudios se centran en la edad escolar, se ha reportado que la mayoría de los niños postoperados de craneosinostosis simple tienen un Coeficiente Intelectual dentro de los parámetros normales ${ }^{37-39}$, sin embargo, pueden presentar déficits en diferentes áreas, entre las que se encuentran: atención, planeación, velocidad de procesamiento, lenguaje $\mathrm{e}^{37}$, habilidades visoespaciales ${ }^{37-38}$, inhibición ${ }^{40}$, así como dificultades en el aprendizaje, en porcentajes variables $(14 \% \text { a } 50 \%)^{37,38,41}$ (Tabla 4 ).

\section{Discusión}

De acuerdo con la literatura, en los primeros tres años de vida, las principales afectaciones que presentan los pacientes con craneosinostosis simple se encuentran en las áreas psicomotora y cognoscitiva (e.g. ${ }^{25}$ ), después de los tres años son frecuentes las alteraciones en el lenguaje y finalmente en la etapa escolar se reportan alteraciones en el funcionamiento ejecutivo, en el lenguaje y problemas de aprendizaje (e.g. $\left.{ }^{37}\right)$. 
Tabla 2. Estudios realizados sobre el funcionamiento cognoscitivo durante los primeros 3 años de vida en pacientes con craneosinostosis simple que realizan evaluaciones pre y postquirúrgicas

\begin{tabular}{|c|c|c|c|c|c|c|}
\hline Autores & $\begin{array}{l}\text { n de pacientes/ } \\
\text { diagnóstico/ } \\
\text { controles }\end{array}$ & $\begin{array}{c}\text { Edad en } \\
\text { evaluación } \\
\text { prequirúrgica }\end{array}$ & $\begin{array}{c}\text { Edad en } \\
\text { evaluación } \\
\text { postquirúrgica }\end{array}$ & Instrumentos & $\begin{array}{c}\text { Resultados } \\
\text { prequirúrgicos }\end{array}$ & $\begin{array}{c}\text { Resultados } \\
\text { postquirúrgicos }\end{array}$ \\
\hline $\begin{array}{l}\text { Cohen } \\
\text { et al. }{ }^{25}\end{array}$ & $\begin{array}{l}\text { 22/prequirúrgicos } \\
10 \text { sagital } \\
7 \text { coronal } \\
5 \text { metópica } \\
15 \text { postquirúrgico }\end{array}$ & $\begin{array}{l}2 \text { meses antes } \\
\text { de la cirugía }\end{array}$ & $\begin{array}{l}1 \text { año después } \\
\text { de la cirugía }\end{array}$ & BSID-II & $\begin{array}{c}\text { DES en los } \\
\text { índices mental } \\
\text { y motor }\end{array}$ & $\begin{array}{l}\text { Índice motor } \\
\text { mejora }\end{array}$ \\
\hline $\begin{array}{l}\text { Bellew } \\
\text { et al. }{ }^{24}\end{array}$ & 28/escafocefalia & 6,9 meses & $\begin{array}{c}15,2 ; 46,5 ; 49,9 \\
\text { y } 60,1 \text { meses }\end{array}$ & $\begin{array}{c}\text { Griffiths Mental } \\
\text { Development } \\
\text { Scales }\end{array}$ & $\begin{array}{l}\text { Deficiencias en } \\
\text { locomoción en } \\
\text { comparación } \\
\text { con el grupo } \\
\text { control }\end{array}$ & $\begin{array}{l}\text { Locomoción } \\
\text { mejora }\end{array}$ \\
\hline $\begin{array}{l}\text { Starr } \\
\text { et al. }\end{array}$ & $\begin{array}{l}\text { 168/CS } \\
86 \text { escafocefalia } \\
35 \text { trigonocefalia } \\
20 \text { plagiocefalia } \\
\text { derecha } \\
16 \text { plagiocefalia } \\
\text { izquierda } \\
11 \text { plagiocefalia } \\
\text { posterior }\end{array}$ & Sin especificar & 18 meses & BSID-II y PLS & $\begin{array}{l}\text { Desempeño } \\
\text { similar o } \\
\text { ligeramente } \\
\text { peor que los } \\
\text { controles }\end{array}$ & $\begin{array}{c}\text { Desempeño } \\
\text { similar o } \\
\text { ligeramente peor } \\
\text { que los controles }\end{array}$ \\
\hline $\begin{array}{l}\text { Da Costa } \\
\text { et al. }{ }^{27}\end{array}$ & $\begin{array}{l}\text { 64/prequirúrgicos } \\
26 \text { escafocefalia } \\
21 \text { trigonocefalia } \\
10 \text { unicoronal } \\
7 \text { multisutural } \\
\text { 44/postquirúrgicos } \\
26 \text { escafocefalia } \\
15 \text { trigonocefalia } \\
7 \text { unicoronal } \\
6 \text { multisutural }\end{array}$ & 4-16 meses & 6-32 meses & BSID-II & $\begin{array}{l}\text { Puntuaciones } \\
\text { más bajas } \\
\text { que las } \\
\text { esperadas por } \\
\text { la población } \\
\text { en los índices } \\
\text { mental y motor }\end{array}$ & $\begin{array}{c}\text { Puntuaciones } \\
\text { más bajas que las } \\
\text { esperadas por la } \\
\text { población en los } \\
\text { índices mental y } \\
\text { motor }\end{array}$ \\
\hline $\begin{array}{l}\text { Kunz } \\
\text { et al. }{ }^{29}\end{array}$ & 25/trigonocefalia & 9,2 meses & $\begin{array}{l}6 \text { y } 12 \text { meses } \\
\text { después de } \\
\text { la cirugía }\end{array}$ & $\begin{array}{l}\text { Cuestionario } \\
\text { estandarizado } \\
\text { que incluía } \\
\text { motricidad gruesa, } \\
\text { coordinación } \\
\text { manual, lenguaje y } \\
\text { función cognitiva }\end{array}$ & $\begin{array}{c}40 \% \text { con } \\
\text { retraso ligero } \\
\text { y moderado, } \\
12 \% \text { retraso } \\
\text { severo en áreas } \\
\text { motoras y de } \\
\text { lenguaje }\end{array}$ & $\begin{array}{c}\text { A los } 6 \text { meses } \\
\text { el } 23 \% \text { y a los } \\
12 \text { meses el } \\
30 \% \text { aún tenían } \\
\text { déficits aparentes }\end{array}$ \\
\hline
\end{tabular}

CS: Craneosinostosis simple; BSID-I: Escalas Bayley del Desarrollo Infantil-I BSID-II: Escalas Bayley del Desarrollo Infantil II; DES: Diferencias estadísticamente significativas; PLS: Preschool Language Scale. 
Tabla 3. Estudios que mencionan el funcionamiento cognoscitivo en edad preescolar de pacientes con craneosinostosis simple

\begin{tabular}{|c|c|c|c|c|}
\hline Autores & $\begin{array}{l}\text { n de casos/ } \\
\text { diagnóstico }\end{array}$ & Edades de evaluación & Instrumentos & Resultados \\
\hline $\begin{array}{l}\text { Shipster } \\
\text { et al. }{ }^{32}\end{array}$ & 76/escafocefalia & $\begin{array}{l}9 \text { meses- } 15 \text { años } \\
\text { Media: } 4 \text { años } 7 \text { meses }\end{array}$ & $\begin{array}{l}\text { 2-3 años y medio: BSID-II, PLS } \\
\text { tercera edición y Phonological } \\
\text { Assessment of Children's Speech. } \\
3 \text { años y medio a } 7 \text { años: Escala } \\
\text { Wechsler, PLS tercera edición, } \\
\text { British Picture Vocabulary } \\
\text { Scale, Reenfrew Word Finding } \\
\text { Vocabulary Scale y Edinburg } \\
\text { articulation test }\end{array}$ & $\begin{array}{l}37 \% \text { con déficit en el } \\
\text { habla o lenguaje. Del } \\
37 \% \text {, el } 71 \% \text { cumplió } \\
\text { los criterios de un } \\
\text { trastorno específico } \\
\text { del lenguaje. El } 46 \% \\
\text { con déficit en la } \\
\text { lectura }\end{array}$ \\
\hline $\begin{array}{l}\text { Becker } \\
\text { et al. } .^{33}\end{array}$ & $\begin{array}{l}\text { 214/ } \\
70 \text { escafocefalia } \\
37 \text { trigonocefalia } \\
59 \text { unicoronal } \\
19 \text { multisutural } \\
9 \text { plagiocefalia } \\
\text { posterior }\end{array}$ & $\begin{array}{c}\text { Pacientes con y sin } \\
\text { cirugía } \\
\text { (4 meses a 20,1 años) }\end{array}$ & $\begin{array}{l}\text { Revisión de documentación } \\
\text { de un examen de lenguaje } \\
\text { estandarizado durante la cita } \\
\text { de revisión. Child Behavioral } \\
\text { Checklist. BSID-I, BSID- } \\
\text { II, Vineland y Cattelle. El } \\
\text { desempeño escolar se evaluó si } \\
\text { se reportaba un problema de } \\
\text { aprendizaje o asistencia a clases } \\
\text { de educación especial }\end{array}$ & $\begin{array}{l}\text { El } 23 \% \text { con } \\
\text { anormalidades } \\
\text { en el habla. } 44 \% \\
\text { con alteraciones } \\
\text { psicológicas. } 35 \% \text { con } \\
\text { puntuaciones altas } \\
\text { en el Child Behavior } \\
\text { Check List }\end{array}$ \\
\hline $\begin{array}{l}\text { Mendonca } \\
\text { et al. }{ }^{35}\end{array}$ & 20/trigonocefalia & $\begin{array}{l}\text { Evaluación del } \\
\text { lenguaje } \\
\text { a los } 3 \text { y } 5 \text { años }\end{array}$ & No especificado & $\begin{array}{l}30 \% \text { con deficiencias } \\
\text { en el lenguaje }\end{array}$ \\
\hline $\begin{array}{l}\text { Starr } \\
\text { et al. }{ }^{36}\end{array}$ & $\begin{array}{l}209 / \\
94 \text { escafocefalia } \\
50 \text { trigonocefalia } \\
52 \text { unicoronal } \\
10 \text { plagiocefalia } \\
\text { posterior }\end{array}$ & 3 años & BSID-II y PLS tercera edición & $\begin{array}{l}\text { Niños con CS } \\
\text { con puntuaciones } \\
\text { más bajas en } \\
\text { todas las medidas, } \\
\text { estadísticamente } \\
\text { significativas en } \\
\text { comparación con el } \\
\text { grupo control }\end{array}$ \\
\hline $\begin{array}{l}\text { Korpilahti } \\
\text { et al. }{ }^{34}\end{array}$ & $\begin{array}{l}61 / 32 \text { CS } \\
20 \text { escafocefalia } \\
12 \text { otros tipos } \\
29 \begin{array}{l}\text { plagiocefalia } \\
\text { posterior } \\
\text { posicional }\end{array}\end{array}$ & 3 años & $\begin{array}{l}\text { Cuestionario para padres de } \\
\text { evaluación del lenguaje. The } \\
\text { Reynell Developmental Language } \\
\text { Scales III. Renfrew Naming Task, } \\
\text { MacArthur Communicative } \\
\text { Development Inventories y } \\
\text { Observación de los niños en } \\
\text { una situación de juego semi- } \\
\text { estructurada }\end{array}$ & $\begin{array}{l}\text { El } 30 \% \text { con retraso } \\
\text { mínimo y el } 21 \% \text { con } \\
\text { retraso severo del } \\
\text { lenguaje }\end{array}$ \\
\hline
\end{tabular}

CS: Craneosinostosis simple; BSID-I: Escalas Bayley del Desarrollo Infantil-I BSID-II: Escalas Bayley del Desarrollo Infantil II; DES: Diferencias estadísticamente significativas. PLS: Preschool Language Scale.

En los primeros dos años de vida, existen diversos cambios en la motricidad de los niños, Iverson $^{42}$ señala que el niño interactúa de distinta forma con el ambiente, y tiene un impacto en el desarrollo de habilidades y experiencias que juegan un rol importante en la comunicación y el len- guaje. Esta autora menciona que la adquisición de habilidades motoras permite que el niño presente habilidades que son relevantes para la adquisición del lenguaje, antes de que sean utilizadas para ese propósito. Por lo anterior, el retraso psicomotor reportado durante los primeros tres años de vida, 
Tabla 4. Estudios que mencionan el funcionamiento cognoscitivo en edad escolar de pacientes con craneosinostosis simple

\begin{tabular}{|c|c|c|c|c|}
\hline Autores & $\begin{array}{l}\text { n de caso/ } \\
\text { diagnóstico }\end{array}$ & $\begin{array}{l}\text { Edades de } \\
\text { evaluación }\end{array}$ & Instrumento & Resultados \\
\hline $\begin{array}{l}\text { Magge } \\
\text { et al. }^{38}\end{array}$ & 16/escafocefalia & $\begin{array}{l}\text { 6-16 años } \\
\text { (media } \\
10,3 \text { años) }\end{array}$ & $\begin{array}{l}\text { WISC-III, Wide Range Achievement } \\
\text { Test- Revised, Connor's Continuous } \\
\text { Performance Test, Wisconsin Card } \\
\text { Sorting Test, Developmental Test of } \\
\text { Visual-Motor Integration y Vineland } \\
\text { Adaptative Behavior Scales }\end{array}$ & $\begin{array}{l}\text { CI dentro de la norma. El } 50 \% \text { de la } \\
\text { población tuvo algún problema de apren- } \\
\text { dizaje }\end{array}$ \\
\hline $\begin{array}{l}\text { Becker } \\
\text { et } \mathrm{al.}^{33}\end{array}$ & $\begin{array}{l}\text { 214/ } \\
70 \text { escafocefalia } \\
37 \text { trigonocefalia } \\
59 \text { unicoronal } \\
19 \text { multisutural } \\
9 \text { plagiocefalia } \\
\text { posterior }\end{array}$ & $\begin{array}{l}\text { Incluidos } \\
\text { pacientes } \\
\text { operados y } \\
\text { no operados } \\
\text { (4 meses a } \\
20,1 \text { años) }\end{array}$ & $\begin{array}{l}\text { Revisión de documentación de un } \\
\text { examen de lenguaje estandarizado } \\
\text { durante la cita de revisión. Child } \\
\text { Behavioral Checklist. BSID-I, } \\
\text { BSID-II, Vineland y Cattelle. } \\
\text { La función escolar se evaluó si } \\
\text { se reportaba un problema de } \\
\text { aprendizaje o la presencia en un } \\
\text { salón de educación especial }\end{array}$ & $\begin{array}{l}47 \% \text { de la muestra de más de } 5 \text { años } \\
\text { presentó déficits cognitivos y } \\
\text { de comportamiento }\end{array}$ \\
\hline $\begin{array}{l}\text { Chieffo } \\
\text { et al. }\end{array}$ & $\begin{array}{l}65 / \\
35 \text { escafocefalia } \\
30 \text { plagiocefalia }\end{array}$ & $\begin{array}{l}\text { 9,2-16,10 } \\
\text { Edad media } \\
\text { de cirugía } \\
\text { 7,2 meses }\end{array}$ & $\begin{array}{l}\text { Edinburg Inventory. Purdue } \\
\text { Pegboard Test. WISC-R. Batteria } \\
\text { per la Valutazionedella Dislessia } \\
\text { e Disortografia Evolutiva. } \\
\text { Developmental Test of Visual } \\
\text { Motor and Perceptual Integration. } \\
\text { Figura de Rey. Bells Cancellation } \\
\text { Test. Torre de Londres. La } \\
\text { función escolar fue evaluada } \\
\text { por la presencia de trastornos } \\
\text { de aprendizaje o retraso en el } \\
\text { desarrollo }\end{array}$ & $\begin{array}{l}\text { CI promedio: } 43 \% \text {. } \\
\text { Escafocefalia: deficiencias visoespaciales, } \\
\text { visoconstructivas y recuperación de mate- } \\
\text { rial visual (7\%), déficit en atención selectiva } \\
\text { y sostenida }(17 \%) \text {, alteraciones en la escri- } \\
\text { tura }(14 \%) \text {, lectura lenta }(6 \%) \text {, déficit en la } \\
\text { comprensión lectora ( } 17 \%) \text {. } \\
\text { Plagiocefalia: déficit en fluidez verbal ( } 30 \%) \text {, } \\
\text { comprensión del lenguaje ( } 17 \%) \text {, en deno- } \\
\text { minación }(7 \%) \text {, memoria de trabajo, coordi- } \\
\text { nación ojo mano, viso percepción y atención } \\
\text { (10\%), déficit en velocidad y comprensión } \\
\text { lectora (13\%) y en la escritura (7\%) }\end{array}$ \\
\hline $\begin{array}{l}\text { Speltz } \\
\text { et al. }{ }^{41}\end{array}$ & $\begin{array}{l}182 / \\
76 \text { escafocefalia } \\
48 \text { trigonocefalia } \\
46 \text { unicoronal } \\
12 \text { plagiocefalia } \\
\text { posterior }\end{array}$ & $\begin{array}{l}6,9-9,5 \text { años } \\
\text { (media } 7-5)\end{array}$ & $\begin{array}{l}\text { WISC-IV, Wide Range } \\
\text { Achievement Test, cuartaedición, } \\
\text { Word Reading Efficiency, } \\
\text { Comprenhensive Test of } \\
\text { Phonological Processing }\end{array}$ & $\begin{array}{l}\text { Puntuaciones más bajas que los controles } \\
\text { en CI total y habilidades aritméticas. } \\
40 \% \text { de la muestra tuvo problemas de } \\
\text { aprendizaje }\end{array}$ \\
\hline $\begin{array}{l}\text { Collett } \\
\text { et al. }{ }^{40}\end{array}$ & 175 & $\begin{array}{c}6,9-9,5 \\
(\text { media } \\
7,5 \text { años })\end{array}$ & $\begin{array}{l}\text { Test of Everyday Attention in } \\
\text { Children, Test de inhibición de } \\
\text { Developmental Neuropsychological } \\
\text { Assessment, indice de memoria de } \\
\text { trabajo del WISC-IV, subescala de } \\
\text { atención de Achenbach System of } \\
\text { Empirically Based Assessment y } \\
\text { el Behavioral Rating Inventory of } \\
\text { Executive Function }\end{array}$ & $\begin{array}{l}\text { Las medidas de inhibición y atención } \\
\text { dividida presentaron DES en comparación } \\
\text { con un grupo de niños sin craneosinostosis }\end{array}$ \\
\hline $\begin{array}{l}\text { Bellew } \\
\text { et al. }{ }^{39}\end{array}$ & $\begin{array}{l}91 / \\
47 \text { escafocefalia } \\
15 \text { plagiocefalia } \\
13 \text { trigonocefalia } \\
9 \text { multisutural } \\
7 \text { braquicefalia }\end{array}$ & 10 años & $\begin{array}{l}\text { WISC-IV o la escala Wechsler } \\
\text { abreviada }\end{array}$ & $\begin{array}{l}\text { CI dentro de la norma } \\
\text { CI verbal mayor que el de ejecución }\end{array}$ \\
\hline
\end{tabular}

BSID-I: Escalas Bayley del Desarrollo Infantil-I BSID-II: Escalas Bayley del Desarrollo Infantil II; DES: Diferencias estadísticamente significativas. CI: Coeficiente intelectual. 
podría ser una causa del retraso en la adquisición del lenguaje que se observa en los niños con craneosinostosis simple en edades posteriores.

Se sabe que las dificultades de aprendizaje, sobre todo la dislexia, tiene como base problemas de lenguaje y que éstos pueden ser evidentes desde los primeros años de vida manifestándose como retraso en su adquisición, dificultad para procesar el sonido de las palabras ${ }^{43,44} \mathrm{y}$ un vocabulario deficiente ${ }^{45}$. Algunos reportes en niños con craneosinostosis simple mencionan problemas en la adquisición del lenguaje, problemas de articulación y de abstracción y posiblemente sean estas deficiencias las que podrían estar condicionando las dificultades en el aprendizaje reportadas en etapa escolar.

En lo que respecta al desarrollo psicomotor, existen investigaciones que refieren que un pobre control postural en los primeros meses de edad se correlaciona con dificultad en la resolución de problemas y en la atención a los 2 años $^{46}$ y que el desarrollo psicomotor grueso en la infancia predice el desempeño cognitivo en la edad escolar, en especial en tareas que involucran la memoria de trabajo y la velocidad de procesamiento ${ }^{47}$. Aunado a lo anterior, el retraso en el desarrollo psicomotor y en el lenguaje entre 9 y 18 meses se asocia con déficit de atención ${ }^{48}$. Estos hallazgos parecen indicar que las deficiencias en el desarrollo psicomotor que presentan los niños con craneosinostosis simple durante los primeros años de edad, podrían causar alteraciones a largo plazo en distintos procesos cognoscitivos.

\section{Conclusiones}

Los reportes de las diferentes evaluaciones neuropsicológicas realizadas a niños con craneosinostosis simple se caracterizan por presentar hallazgos contradictorios, lo cual muy probablemente se debe a la heterogeneidad en la metodología empleada (diferentes instrumentos, tiempos de evaluación, características de las muestras). Lo anterior ha provocado dificultad para generar un perfil neuropsicológico consistente de esta pobla- ción. La carencia de grupos controles y muestras pequeñas, en la mayoría de los estudios no permiten realizar conclusiones que se puedan generalizar a todos los pacientes con la patología. Toda esta variabilidad obstaculiza llegar a conclusiones únicas, no obstante, pueden encontrarse algunas regularidades:

Durante los primeros años de vida es frecuente encontrar retraso en la adquisición del lenguaje (43-45\% de las muestras), retraso cognitivo y psicomotor, con tendencia a la mejoría en las evaluaciones postquirúrgicas: en la etapa preescolar el retraso en el lenguaje y en la etapa escolar trastornos de aprendizaje.

Todo lo anterior en porcentajes variables, reportando alteraciones entre un 14\% hasta un 50\% de las muestras estudiadas, se podría decir que no se reportan, en su mayoría, alteraciones severas, pero las deficiencias encontradas son consistentes en la literatura revisada.

Aunque se mencionan porcentajes variables de alteraciones estructurales y funcionales, así como deficiencias neuropsicológicas, no hay estudios en donde se analice la correlación entre estas dos variables, por lo que hacen falta investigaciones que permitan conocer de una forma más específica la causa de las alteraciones neuropsicológicas y de desarrollo encontradas en esta población.

A pesar de que no todos los pacientes presentan deficiencias en los procesos cognoscitivos, es recomendable realizar un seguimiento del desarrollo neuropsicológico de estos niños, desde la edad de diagnóstico hasta la edad escolar. Además, es necesario que en las evaluaciones se utilicen instrumentos sensibles que ayuden a la identificación de las deficiencias y que analicen todos los procesos neuropsicológicos y en caso de que se identifiquen alteraciones, generar estrategias específicas de intervención para contrarrestar las posibles deficiencias a largo plazo.

Las características heterogéneas de esta población obstaculizan poder subsanar todas las cuestiones metodológicas referidas, no obstante, sería deseable en futuras investigaciones:

- Intentar correlacionar los hallazgos anatómicos y funcionales con los aspectos cognoscitivos. 
- Hacer estudios de seguimiento a largo plazo.

- Poder hacer una propuesta de consenso de una batería estandarizada para cada una de las edades estudiadas, partiendo de cubrir como mínimo aquellas funciones para las que se ha encontrado más consistencia en la literatura.

- Hacer en todos los casos estudios pre y postquirúrgicos.

\begin{abstract}
Resumen
Introducción: La craneosinostosis simple es el cierre prematuro de una o más suturas craneales que provoca crecimiento anormal del cráneo y en algunos casos anormalidades cerebrales estructurales $y / o$ funcionales. Es un tema controversial si estas anormalidades causan el retraso psicomotor y/o cognitivo reportado en estos pacientes. Esta revisión tiene como objetivo abordar los hallazgos encontrados en el desarrollo psicomotor y cognitivo en niños con craneosinostosis, desde el nacimiento hasta la edad escolar, dividiéndolos en tres etapas: nacimiento a 3 años, etapa preescolar y escolar. Método: Se realizó una búsqueda en PubMed del 2000 al 2015, utilizando estas palabras claves: "craniosynostosis and neurodevelopment", "craniosynostosis and neuropsychological", "Craniosynostosis and cognition". Resultados: En las etapas tempranas de la infancia, los niños con craneosinostosis pueden tener deficiencias motoras, de lenguaje y cognitivas; estos déficits están presentes en un porcentaje variable en la etapa preescolar y pueden acompañarse de alteraciones conductuales; deficiencias atencionales, ejecutivas y de aprendizaje, se reportan en la edad escolar. Conclusión: Se reportan alteraciones neuropsicológicas en un porcentaje variable en estos pacientes en todas las etapas del desarrollo, que se van manifestando conforme las demandas del ambiente. Es difícil establecer un perfil neuropsicológico debido a la heterogeneidad de las características de las muestras en los estudios, el tiempo de la evaluación, las funciones estudiadas y los instrumentos utilizados. Es importante realizar un seguimiento longitudinal para conocer el desarrollo de estos niños, así como el hacer un consenso sobre las funciones cognitivas a evaluar e instrumentos a utilizar para cada edad.
\end{abstract}

Palabras clave: craneosinostosis simple, deficiencias cognitivas, neuropsicología.

\section{Referencias bibliográficas}

1. Aviv RI, Rodger E, Hall CM. Craniosynostosis. Clin Radiol 2002; 57: 93-102.

2. Van Veelen-Vincent ML, Mathijssen I, Arnaud E, Renier D. Non-syndromic Craniosynostosis. En Lumenta CB, Di Rocco C, Haase J, Mooij JJA, editores. Neurosurgery (European Manual of medicine). Berlín: Springer-Verlag; 2009. p. 501-21.

3. FearonJ. Evidence-Based Medicine: Craniosynostosis. Plast Reconstr Surg 2014; 133: 1261-75.

4. Flores L. Avances en craneosinostosis. Rev Mex Neuroci 2003; 4: 63-74.

5. Kabbani H, Raghuveer TS. Craniosynostosis. Am Fam Physician 2004; 69: 2863-70.

6. Renier D, Lajeunie E, Catala M, Arnaud E, Mar- chac D. Craneoestenosis. EMC Pediatría 2008; 43: $1-19$.

7. Cohen MM Jr. Craniosynostosis: Diagnosis, Evaluation, and Management. Nueva York: Raven Press; 1986.

8. Jones M. Terminology and classification of craniosynostosis. En Mooney MP, Siegel MI, editores. Understanding Craniofacial Anomalies: The etiopathogenesis of Craniosynostoses and Facial Clefting. Wiley-Liss: Nueva York; 2002. p. 11-15.

9. Carmel PW, Luken MG, Ascherl GF Jr. Craniosynostosis: computed tomographic evaluation of skull base and calvarial deformities and associated intracranial changes. Neurosurgery 1981; 9: 366-72.

10. Magge KT, Magge SN, Keating, RF, Myseros JS, Boyajian, MJ, Sauerhammer TM, et al. Incidental 
Findings on Preoperative Computed Tomography for Nonsyndromic Single Suture Craniosynostosis. J Craniofac Surg 2014; 25: 1327-30.

11. Aldrige K, Kane AA, Marsh JL, Panchal J, Boyadjiev SA, Yan P, et al. Brain morphology in non syndromic unicornonal craniosynostosis. Anat Rec A Discov Mol Cell Evol Biol 2005; 285: 690-8.

12. Hukki A, Koljonen V, Karppinen A, Valanne L, Leikola J. Brain anomalies in 121 children with non-syndromic single suture craniosynostosis by MR imaging. Eur J Paediatr Neurol 2012; 16: 671-5.

13. Cohen SR, Persing JA. Intracranial pressure in single-suture craniosynostosis. Cleft Palate Craniofac J 1998; 35: 194-6.

14. Tamburrini G, Caldarelli M, Massimi L, Santini P, Di Rocco C. Intracranial pressure monitoring in children with single suture and complex craniosynostosis: a review. Childs Nerv Syst 2005; 21: 913-21.

15. David LR, Genecov D, Camastrea A, Wilson J, Argenta L. Positron emission tomography studies confirm the need for early surgical intervention in patients with single suture craniosynostosis. J Craniofac Surg 1999; 10: 38-42.

16. Iqbal JB, Hockley AD, Wake MJC, Goldin JH. Transcranial Doppler sonography in craniosynostosis. Childs Nerv Syst 1994; 10: 259-363.

17. Beckett JS, Brooks ED, Lacadie C, Vander Wyk B, Jou RJ, Steinbacher DM, et al. Altered brain connectivity in sagittal craniosynostosis. J Neurosurg Pediatr 2014; 13: 690-8.

18. Ricci D, Vasco G, Baranello G, Salerni A, Amante R, Tamburrini G, et al. Visual function in infants with non-syndromic craniosynostosis. Dev Med Child Neurol 2007; 49: 574-6.

19. Vasco G, Baranello G, Ricci D, Salerni A, Tamburrini G, Amant R, et al. Longitudinal assessment of visual development in non-syndromic craniosynostosis: a 1-year pre- and post-surgical study. Arch Dis Child 2008; 93: 932-5.

20. Hashim PW, Brooks ED, Persing JA, Reuman H, Naples A, Travieso R, et al. Direct brain recordings reveal impaired neural function in infants with single-suture craniosynostosis: A future modality for guiding management? J Craniofac Surg 2015; 26: $60-1$.
21. Panchal J, Amirsheybani H, Gurwithc R, Cook V, Francel P, Neas B, et al. Neurodevelopment in children with single-suture craniosynostosis and plagiocephaly without synostosis. Plast Reconstr Surg 2001; 108: 1492-8.

22. Thot K, Collett B, Kapp-Simon KA, Cloonan YK, Gaither R, Cradock MM, et al. Memory and response inhibition in young children with singlesuture craniosynostosis. Child Neuropsychology 2007; 26: 1-14.

23. Warschausky S, Angobaldo J, Kewman D, Buchman S, Muraszko KM, Azengart A. (2005). Early Development of Infants with Untreated Metopic Craniosynostosis. Plast Reconstr Surg 2005; 115: 1518-23.

24. Bellew M, Chumas P, Mueller R, Liddington M, Russell J. Pre-and postoperative developmental attainment in sagittal synostosis. Arch Dis Child 2005; 90: 346-50.

25. Cohen SR, Cho DC, Nichols SL, Cross KP, Burstein FD. American Society of maxillofacial surgeons outcome study: preoperative and postoperative neurodevelopmental findings in single-suture craniosynostosis. Plast Reconstr Surg 2004; 114: 841-7.

26. Da Costa AC, Anderson VA, Savarirayan R, Wrennall JA, Chong DK, Holmes AD, et al. Neurodevelopmental functioning of infants with untreated single-suture craniosynostosis during early infancy. Childs Nerv Syst 2012; 28: 869-77.

27. Da Costa AC, Anderson VA, Holmes AD, Lo P, Wray AC, Chong DK, et al. Longitudinal study of the neurodevelopmental characteristics of treated and untreated non syndromic craniosynostosis in infancy. Childs Nerv Syst 2013; 29: 985-95.

28. Kapp-Simon KA, Leroux B, Cunningham M, Speltz ML. Multisite Study of Infants With SingleSuture Craniosynostosis: Preliminary Report of Presurgery Development. Cleft Palate Craniofac J. 2005; 42: 377-84.

29. Kunz M, Lehner M, Heger A, Armbruster L, Weigand $\mathrm{H}$, Mast $\mathrm{G}$, et al. Neurodevelopmental and esthetic results in children after surgical correction of metopic suture synostosis: a single institutional experience. Childs Nerv Syst 2014; 30: 1075-82.

30. Starr JR, Kapp-Simon KA, Cloonan YK, Collett BR, Cradock MM, Buono L, et al. Presurgical and 
postsurgical assessment of the neurodevelopment of infants with single-suture craniosynostosis: comparison with controls. J Neurosurg 2007; 107: 103-10.

31. Speltz ML, Kapp-Simon K, Collet B, Keich Y, Gaither R, Cradock MM, et al. Neurodevelopment of infants with single-suture craniosynostosis: presurgery comparisons with case-matched controls. Plast Reconstr Surg 2007; 119: 1874-81.

32. Shipster C, Hearst D, Somerville A, Stackhouse J, Hayward R, Wade A. Speech, language, and cognitive development in children with isolated sagittal synostosis. Dev Med Child Neurol 2003; 45: 34-43.

33. Becker DB, Petersen JD, Kane AA, Caradock MM, Pilgram TK, Marsh JL. Speech, Cognitive, and Behavioral Outcomes in non syndromic craniosynostosis. Plast Reconstr Surg 2004; 116: 400-7.

34. Korpilahti P, Saarinen P, Hukki J. Deficient language acquisition in children with single suture craniosynostosis and deformational posterior plagiocephaly. Childs Nerv Syst 2012; 28: 419-25.

35. Mendonca DA, White N, West E, Doer S, Solanki G, Nihikawa $H$. Is there a relationship between the severity of metopic synostosis and speech and language impairments? J Craniofac Surg 2009; 20: 85-8.

36. Starr JR, Collett BR, Gaither R, Kapp-Simon KA, Cradock MM, Cunningham ML, et al. Multicenter Study of Neurodevelopment in 3-Year-Old Children With and Without Single-Suture Craniosynostosis. Arch Pediatr Adolesc Med 2012; 166: 536-42.

37. Chieffo D, Tamburrini G, Massimi L, Di Giovanni S, Giansanti C, Calderalli M, et al. Long-term neuropsychological development in single-suture craniosynostosis treated early. J Neurosurg Pediatr 2010; 5: 232-7.

38. Magge SN, Westerveld M, Pruzinsky T, Persing JA. Long-Term Neuropsychological Effects of Sagittal Craniosynostosis on Child Development. J Craniofac Surg 2002; 13: 99-104.

39. Bellew M, Chumas P. Long-term developmental follow-up in children with non syndromic craniosynostosis. J Neurosurg Pediatr 2015; 16: 445-51.

40. Collett BR, Kapp-Simon KA, Wallace E, Cradock MM, Buono L, Speltz ML. Attention and executive function in children with and without singlesuture craniosynostosis. Child Neuropsychol 2015; 18: 1-16.

41. Speltz ML, Collett BR, Wallace ER, Starr JR, Cradock MM, Buono L, et al. Intellectual and academic functioning of school-age children with single-suture craniosynostosis. Pediatrics 2015; 135: e615-e623.

42. Iverson JM. Developing language in a developing body: the relationship between motor development and language development. J Child Lang 2010; 37: 229-61.

43. Gilger JW, Wilkins MA. Atypical neurodevelopmental variation as a basis for learning disorders. En Sillman ER, Stone CA, editores. Brain, behavior, and learning in language and reading disorders. Nueva York: The Gilford Press; 2008. p. 7-40.

44. Lyytinen H, Aro M, Wklund K, Erskine J, Guttorm T, Laakso ML, et al. The development of children at familial risk for dyslexia: Birth to early school age. Ann Dyslexia 2004; 54: 184-220.

45. Dlouha O. Late talkers: Children with developmental dysphasia (longitudinal follow-up). EnReed MA, ed. Children and language: development, impairment and training. Nueva York: Nova Science Publishers, Inc; 2009. p. 243-65.

46. Wijnroks L, van Veldhoven N. Individual differences in postural control and cognitive development in preterm infants. Infant Behav Dev 2003; 26: 14-26.

47. Piek JP, Dawson L, Smith LM, Gasson N. The role of early fine and gross motor development on later motor and cognitive ability. Hum Mov Sci 2008; 27: 668-81.

48. Gurevitz M, Geva R, Varon M, Leitner Y. Early markers in infants and toddlers for development of ADHD. J Atten Disord 2014; 18: 14-22.
Correspondencia:
Julieta Moreno Villagómez
Avenida de los Barrios Número 1, Colonia Los
Reyes Iztacala Tlalnepantla, Estado de México, C.P. 54090
Teléfono: 525556231333 ext 39730
Fax: 56907604
E-mail: julietamoreno27@gmail.com 